\title{
Heilung in nur acht Wochen für das Gros der Patienten
}

Zur Therapie der Hepatitis C steht mit Glecaprevir/Pibrentasvir (Marviret ${ }^{\circledR}$ ) eine weitere neue Therapieoption zur Verfügung, die therapienaiven Patienten ohne Zirrhose innerhalb von acht Wochen eine nahezu $100 \%$ ige Heilungschance offeriert. Unbehandelte Patienten ohne fortgeschrittene Leberkomplikationen machen heute den Großteil der Hepatitis-C-Patienten aus, erläuterte Professor Heiner Wedemeyer, Medizinische Hochschule Hannover $(\mathrm{MHH})$. Für sie ist kurzes und einfaches Therapieregime vorteilhaft. Mit dem Protease-Inhibitor Glecaprevir und dem NS5A-Hemmer Pibrentasvir (100 mg/40 mg) hat die Firma Abbvie eine pangenotypisch wirksame Fixkombination zweier neuer Wirkstoffe entwickelt. Das Medikament ist gegen alle HCV-Genotypen wirksam, unabhängig von Alter,
Geschlecht, BMI, Viruslast oder Resistenzlage. Wedemeyer berichtete die Daten des Studienprogramms ENDURANCE bei nicht vorbehandelten HCV-Patienten ohne Zirrhose. Die Heilungsraten nach achtwöchiger Therapie lagen über alle Genotypen bei $97 \%$ und beim in Deutschland häufigsten Genotyp 1 bei $99 \%$. „Wir können heute im Prinzip alle HepatitisC-Patienten heilen", so Wedemeyer. In der MHH habe man bei 800 bis 900 Patienten nur drei echte Therapieversager gesehen.

Wie Studien bei speziellen Patientengruppen zeigen, wirkt Glecaprevir/Pibrentasvir mit gleich hoher Zuverlässigkeit bei Patienten mit Niereninsuffizienz, HIV-Koinfektion oder Opiatsubstitutionstherapie. Auch bei nicht vorbehandelten Patienten mit kompensierter Leberzirrhose liegen die Heilungsraten im Be- reich von $100 \%$. Allerdings muss dazu die Therapie über zwölf Wochen durchgeführt werden. Eine weitere besondere Gruppe stellen HCV-Patienten mit Infektionen durch den in Deutschland zweithäufigsten Genotyp 3 dar, die mit einer schnellen Krankheitsprogression assoziiert sind und als schwierig zu behandeln gelten. Auch bei ihnen lassen sich, sofern keine Zirrhose vorliegt, hohe Heilungsraten mit einer achtwöchigen Therapie erzielen, so Wedemeyer. Bei Zirrhose wird eine zwölfwöchige Behandlung empfohlen, bei Vorbehandlung eine 16-wöchige.

Die Therapie mit Glecaprevir/Pibrentasvir sieht einmal täglich die Einnahme von drei Tabletten vor. Die Verträglichkeit ist gut, häufigste Nebenwirkungen sind Kopfschmerzen und Müdigkeit. Im Studienprogramm haben unter $2.265 \mathrm{~Pa}$ tienten nur $0,4 \%$ die Therapie abgebrochen.

Dr. med. Dirk Einecke

Launch-Pressekonferenz „Don't look back: Auf dem Weg in ein neues Leben ohne Hepatitis C: Die neue Therapiegeneration Maviret ${ }^{\oplus ",}$ Wiesbaden, 18.8.2017; Veranstalter: Abbvie

\section{Probleme im Bauch, nicht im Kopf}

Plötzliche Bauchschmerzen, Anfälle von Übelkeit und teilweise heftiger Durchfall in Stresssituationen, so ergeht es vielen Frauen in Deutschland, zu einem etwas geringeren Anteil leiden auch Männer darunter. Organisch werden meist keine Befunde festgestellt, die die Beschwerden erklären könnten. Damit liegt für sie die Diagnose funktionelle Magen-DarmStörung wie Reizmagen oder Reizdarm nahe. Dabei handelt es sich um Krankheitsbilder, für die diagnostisch keine organischen Ursachen sichtbar sind. Bei der Untersuchung werden von den Ärzten daher Geschwüre, physische Anomalien im Magen-Darm-Trakt oder Nahrungsmittelunverträglichkeiten ausgeschlossen. Was dann als Ursache für die Beschwerden übrig bleibt, ist zum Beispiel eine Überempfindlichkeit der Nerven oder eine Fehlleitung der Nervenimpulse in Magen- oder Darmwand oder eine Störung der Bewegungsabläufe von Magen und Darm. Das Reizdarmsyndrom betrifft 7-25\% der westlichen Bevölke- rung und ist ein Phänomen, das Frauen häufiger betrifft als Männer.

„Viele Patienten, die zu mir in die ernährungstherapeutische Sprechstunde kommen, vermuten bereits eine Verbindung zwischen Stress und Beschwerden im Magen-Darm-Bereich. Wir versuchen dann gemeinsam herauszufinden, ob eine Ernährungsumstellung Linderung verschaffen kann“, sagt Ernährungswissenschaftlerin Amely Brücker. In ihrem beruflichen Alltag hat sie schon viele Reizmagen- und Reizdarmpatienten betreut. Ihre Einschätzung: „Es gibt nicht die eine Betroffene oder den einen klassischen Fall. Dementsprechend gibt es auch nicht das Allheilmittel. Es erfordert eine umfassende Anamnese und viel Geduld, die richtige Therapie für jeden zu finden.“

Für Betroffene ist die Erkenntnis „Ich hab's im Bauch, nicht im Kopf" schon eine große Erleichterung. Dennoch haben Reizmagen- und Reizdarmpatienten oft einen längeren Weg vor sich, bis ein Arzt die richtige Diagnose stellen kann

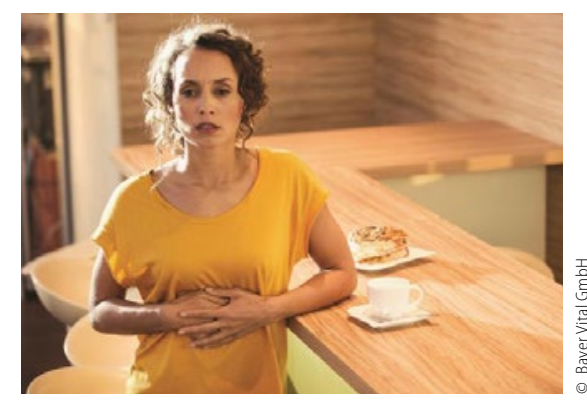

und sie etwas finden, das ihnen Linderung verschafft. Helfen kann zum Beispiel eine Ernährungsumstellung. Unterstützung erhält der Verdauungsapparat bei diesen Erkrankungen auch durch das pflanzliche Mittel Iberogast, eine seit über 55 Jahren bewährte Rezeptur. Die enthaltenen Extrakte aus neun Heilpflanzen wirken nach dem Viele-Ziele-Prinzip. Das bedeutet, sie können unter anderem sowohl die Entspannung als auch die Anregung der Magen- und Darmtätigkeit fördern, an verschiedenen Stellen gleichzeitig ansetzen und so zur umfassenden Linderung der unterschiedlichen $\mathrm{Be}$ schwerden beitragen.

red

Nach Informationen von Bayer Vital GmbH 